\title{
Introducción al análisis de redes
}

\section{Por Antonio Perianes-Rodríguez, Carlos Olmeda-Gómez y Félix de Moya-Anegón}

\begin{abstract}
Resumen: La teoría de redes y el análisis estructural son poco conocidos en nuestra área, tanto en el campo teórico, como metodológico. El actual período de revolución en el análisis de redes que también afecta a la biblioteconomía y documentación debe cambiar este hecho, ya que su aplicación implica un salto cuantitativo y cualitativo en la representación y el análisis de la estructura de todo tipo de dominios científicos, ya sean geográficos, temáticos, institucionales e incluso individuales. Este trabajo tiene por objeto la caracterización de los distintos tipos de redes del mundo real según su tipo y tamaño, esta-

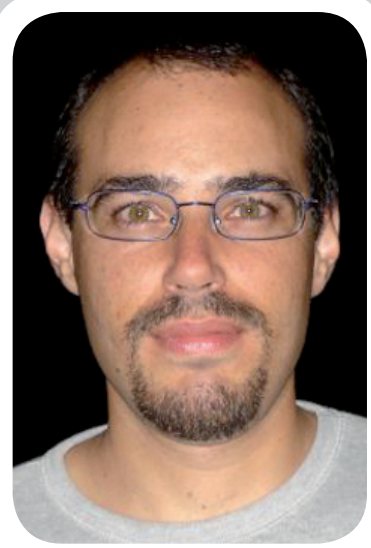

Antonio Perianes-Rodríguez, es profesor ayudante en el Departamento de Biblioteconomía y Documentación de la Universidad Carlos III de Madrid e investigador del grupo SCImago.
\end{abstract}

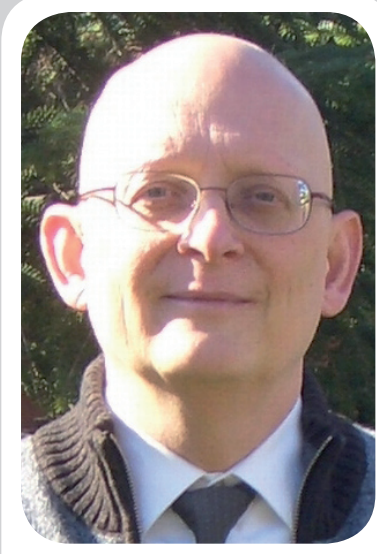

Carlos Olmeda-Gómez, es profesor titular en el Departamento de Biblioteconomía y Documentación de la Universidad Carlos III de Madrid e investigador del grupo SClmago.

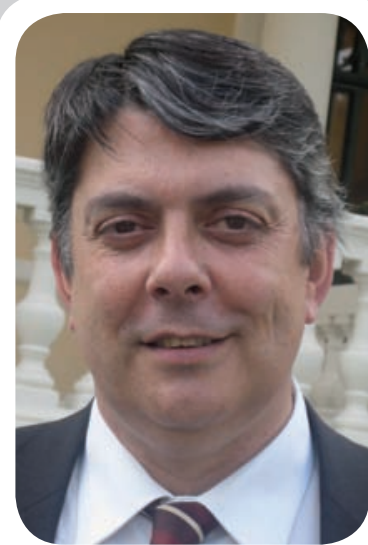

Félix de Moya-Anegón, es catedrático en el Departamento de Biblioteconomía y Documentación de la Universidad de Granada y director del grupo SCImago.

bleciendo un primer criterio para la determinación de redes con posible aplicación en los estudios de biblioteconomía y documentación. En segundo lugar, un análisis de los diversos tipos de redes, a través de las teorías que estudian el comportamiento y la dinámica estructurales, y la influencia de los trabajos de nuestra área para el establecimiento de la explicación más probable para el crecimiento y la evolución de las redes reales.

Palabras clave: Biblioteconomía y documentación, Análisis de redes, Teoría de redes, Investigación científica.

\section{Title: Introduction to network analysis}

Abstract: Despite its long existence and international acceptance, network theory and analysis is a practically unknown approach in Documentation, both theoretically and methodologically speaking. Fortunately, this trend is changing, inasmuch as network theory and analysis may mean a quantitative and qualitative leap forward in the representation and analysis of the structure of all types of scientific domains, whether geographic, thematic or institutional. The extraordinary advances that have taken place in recent years in the study and analysis of complex networks have been made possible by a number of parallel developments. First of all, with computerized data acquisition and handling, large databases can be managed, leading to the emergence of different real network topologies. Secondly, the increase in computing power has made it possible to explore networks with millions of nodes. Thirdly, there is the slow but sure breakdown of boundaries between disciplines. This can be seen by researchers because of their ability to access and use databases that facilitate an understanding of the generic properties of complex networks.

Keywords: Library and information science, Network analysis, Network theory, Scientific research.

Perianes-Rodríguez, Antonio; Olmeda-Gómez, Carlos; Moya-Anegón, Félix de. "Introducción al análisis de redes". En: El profesional de la información, 2008, noviembre-diciembre, v. 17, n. 6, pp. 664-669.

DOI: 10.3145/epi.2008.nov.10

\section{Introducción}

LA TEORÍA DE REDES y el análisis estructural son poco conocidos en nuestra área, tanto en el campo teórico, como metodológico.

El actual período de revolución en el análisis de redes que también afecta a la biblioteconomía y docu- mentación debe cambiar este hecho, ya que su aplicación supone un salto cuantitativo y cualitativo en la representación y el análisis de la estructura de todo tipo de dominios científicos, ya sean geográficos, temáticos, institucionales e incluso individuales (Otte; Rousseau, 2002; Perianes-Rodríguez et al.,
2008; Vargas-Quesada; MoyaAnegón, 2007).

Las redes se encuentran por todas partes, nos rodean, formamos parte de ellas, unas veces como nodos (en nuestras relaciones de parentesco o amistad), en otras ocasiones como enlaces (fluyendo como usuarios entre estaciones o 
aeropuertos). Las redes de comunicación, la World Wide Web (www), el genoma humano, las redes de proteínas, las redes neuronales, las de transportes, las redes sociales, las redes de colaboración científica o las redes terroristas son algunos ejemplos. Incluso el lenguaje que nos sirve para escribir este trabajo es una red, compuesta por palabras unidas por relaciones sintácticas y semánticas (Barabási; Bonabeau, 2003; Barrat et al., 2004; Börner et al., 2005).

\section{"Las redes se encuentran por todas partes, nos rodean, formamos parte de ellas, unas veces como nodos, en otras ocasiones como enlaces"}

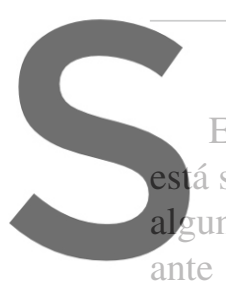

El progreso de esta especialidad siendo tan acelerado que según unos autores nos encontramos ante el surgimiento de una nueva disciplina basada en un nuevo conRegisterefor, freelat lațtps/ hwewkw.scip sofía, la del mundo pequeño (small world).

Pero no debemos olvidar que los extraordinarios avances que han tenido lugar en los últimos años en el estudio y análisis de redes complejas no hubieran sido posibles sin otros procesos paralelos: la adquisición y manipulación de datos por ordenador, que ha permitido manejar voluminosas bases de datos, o el aumento del potencial de computación que ha permitido la exploración de redes con millones de nodos (Albert; Barabási, 2002).

\section{Objetivos}

Este trabajo tiene por objeto la caracterización de los distintos tipos de redes del mundo real según su tipo y tamaño, estableciendo un primer criterio para la determina- ción de redes con posible aplicación en los estudios de biblioteconomía y documentación. En segundo lugar, un análisis de los diversos tipos de redes, a través de las teorías que estudian el comportamiento y la dinámica estructurales, y la influencia de los trabajos de nuestra área para el establecimiento de la explicación más probable para el crecimiento y la evolución de las redes reales. Finalmente, se exponen los principales factores que influyen en el crecimiento y la evolución de redes reales.

\section{Breve reseña histórica}

Se considera que la teoría de redes tuvo su inicio con el matemático suizo Leonhard Euler que planteó el curioso problema de los siete puentes sobre el río Pregel de la ciudad prusiana de Kaliningrado: ¿es posible dar un paseo comenzan-
(Moreno, 1934). Pero el verdadero desarrollo en redes sociales se llevó a cabo con la introducción de medidas destinadas a la obtención de patrones de conexiones sociales que enlazaran conjuntos de actores en psicología para detectar grupos sociales (interrelación de actores) o las posiciones de los actores en la red (detección de actores estructuralmente similares) (Molina, 2001; Rodríguez, 1995).

También fue Moreno el introductor de la primera representación gráfica de una matriz de datos para el análisis de patrones psicológicos, el sociograma (Moreno, 1953). Desde el punto de vista de la abstracción o la información visual, el sociograma presenta ventajas sobre la información meramente numérica o tabular, ya que hace posible transmitir la información estructural de la red de forma sencilla y des-

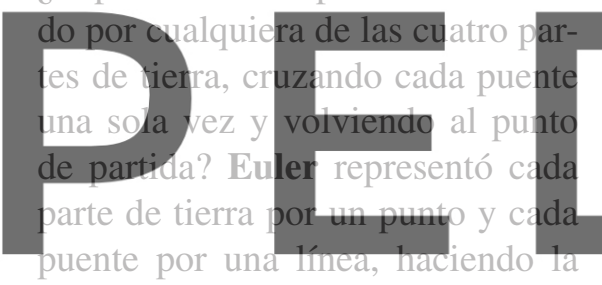

siguiente pregunta: ise puede reco-

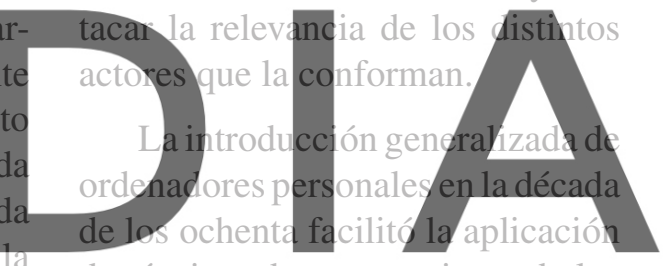

de técnicas de agrupamiento de los

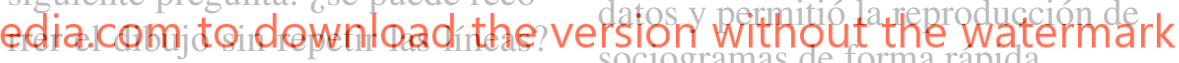
(figura 1).

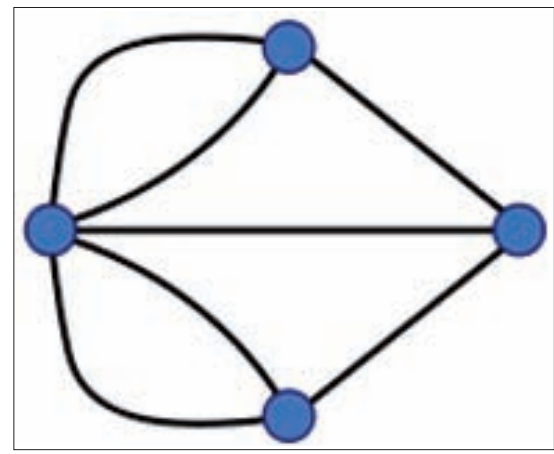

Planteamiento del problema de los puentes de Kaliningrado

Ya en el siglo XX, la teoría de grafos cobró un nuevo impulso gracias a la intervención de especialistas en psiquiatría y antropología social que introdujeron el concepto de análisis de redes sociales en los años treinta del siglo pasado

\section{'Las redes 'reales' se dividen en cuatro categorías según su tipo: sociales, de información, tecnológicas y biológicas"}

\section{Tipos de redes}

Como se ha dicho, las redes son ubicuas. A continuación se revisará de manera sucinta la estructura y diferencias que se dan entre los distintos tipos de redes del mundo real, basadas en los análisis que tratan de explicar y modelar sus propiedades. Las redes "reales" se dividen en cuatro categorías según su tipo. El 

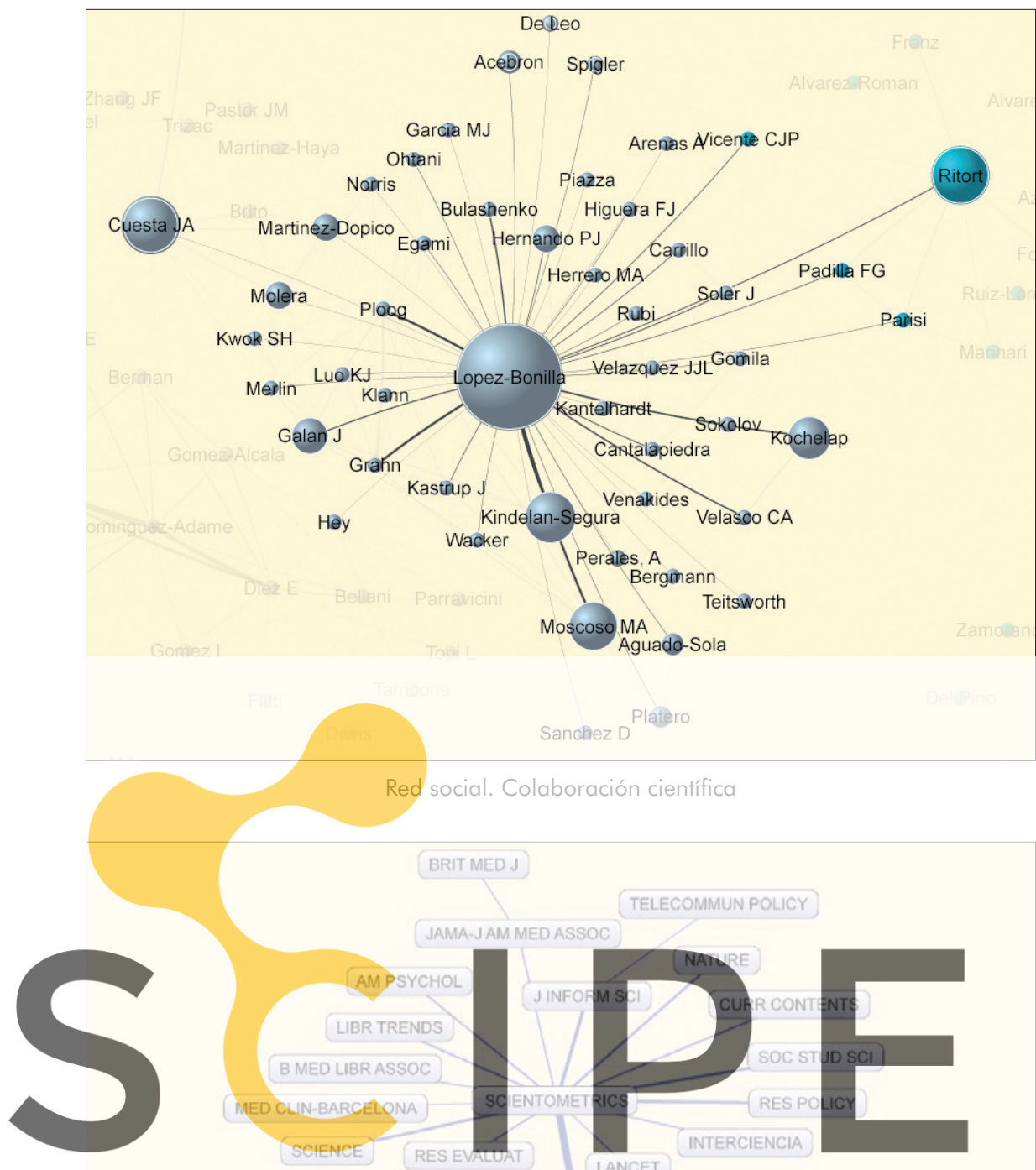

origen de las diferencias entre unas y otras radica en los datos empleados para la elaboración y extracción de las matrices (Newman, 2003):

\subsection{Redes sociales}

Las redes sociales están compuestas por individuos o grupos de individuos con patrones de contactos o interacciones entre ellos. Ejemplos de este tipo de redes son las relaciones de amistad, de negocios entre directivos de empresas, o entre familias a partir de sus matrimonios y descendencia (genealogías).

Al análisis de este tino de redes se asocian a menudo dificultades de imprecisión y subjetividad, debidas al reducido tamaño de las muestras que emplean y a los métodos utilizados para la recogida de datos: generalmente encuestas, cuestionarios o entrevistas.

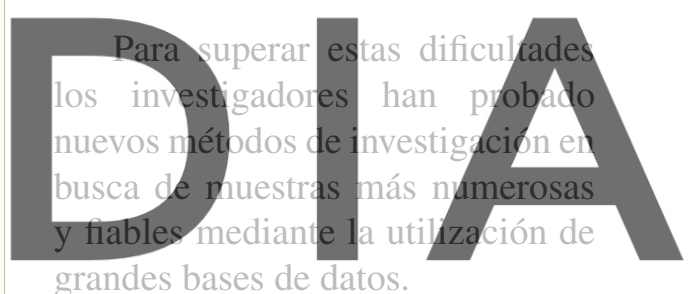

Register for free at https//.www.scipedia.com to download the version without the watermark

\section{BIOL CYBERN}

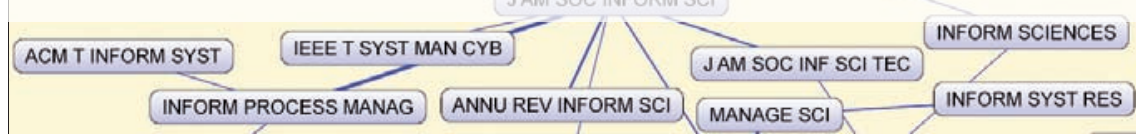

Red de información. Cocitación de revistas

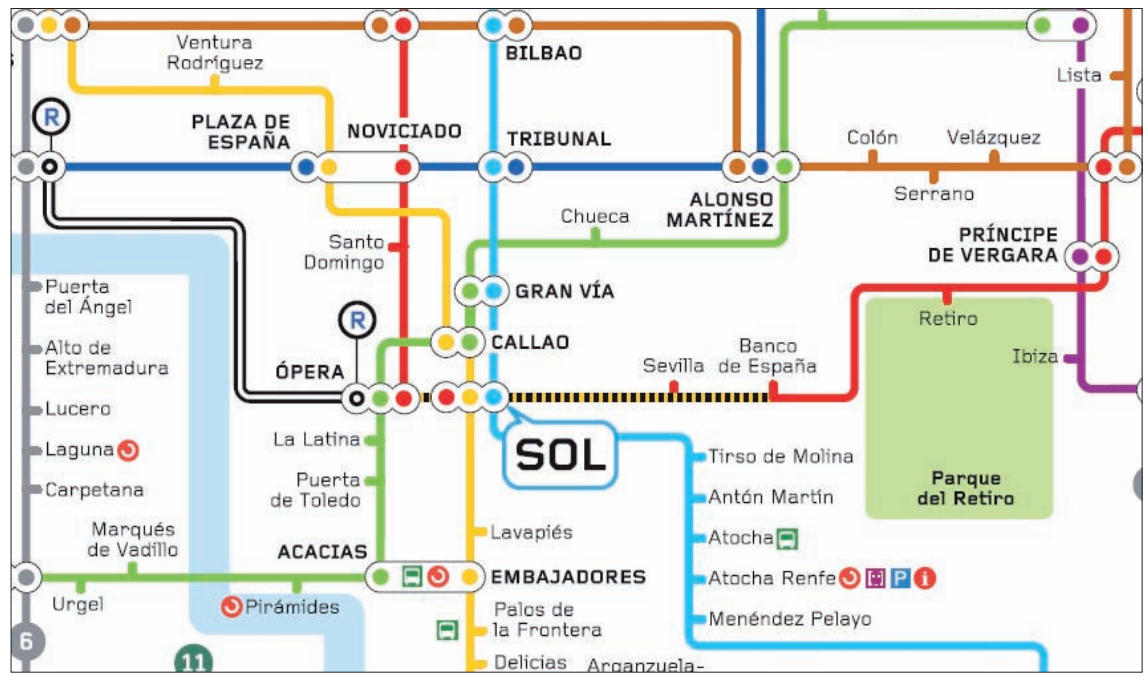

Red tecnológica. Red de metro

Figura 2. Ejemplos de redes según tipo de datos
"Una red de información ampliamente estudiada es la World Wide Web, que contiene páginas informativas que se enlazan a través de hipervínculos"

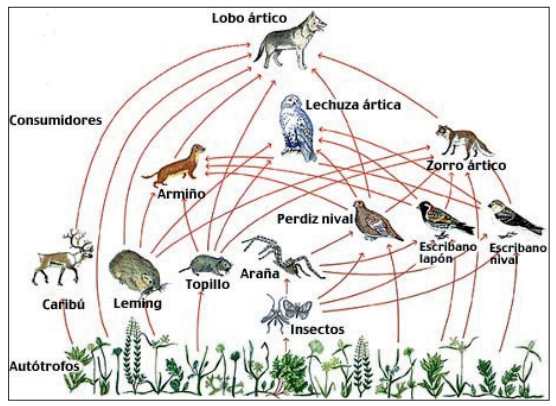

Red biológica. Cadena trófica ártica 


\subsection{Redes de información}

También denominadas redes de conocimiento. El ejemplo clásico de redes reales de esta categoría son las de citas y cocitas de trabajos científicos. Otro ejemplo ampliamente estudiado de redes de información es la World Wide Web (no debe confundirse con la internet física), red que contiene páginas informativas que se enlazan a través de hipervínculos (Faba Pérez et al., 2004; Faba Pérez et al., 2005). Al igual que las redes de citas, en la www también influyen aspectos sociales que trascienden el mero interés informativo de los vínculos.

\subsection{Redes tecnológicas}

Son las redes diseñadas para la distribución de electricidad (energía), agua, gas, las redes de transportes (carreteras, ferrocarril, rutas aéreas), las redes telefónicas (sólo

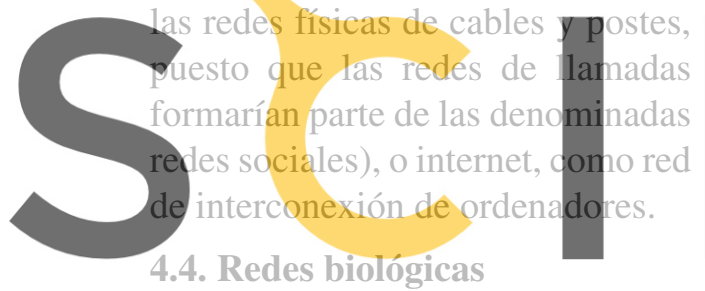

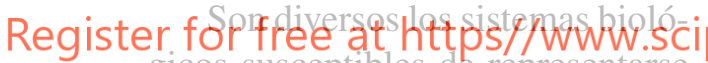
gicos susceptibles de representarse en forma de redes. Las redes de reacciones metabólicas, las redes genéticas, los ecosistemas y cadenas tróficas, las redes neuronales o las vasculares son algunos de los ejemplos de redes biológicas analizadas desde la perspectiva de la teoría de redes. Las redes alimentarias, por ejemplo, pueden ser descritas como un grafo con un conjunto finito de nodos (especies) y un conjunto finito de enlaces que asocian cada uno de esos nodos entre sí. El análisis del grado saliente y entrante (indegree y outdegree) de las redes alimentarias posibilita extraer abstracciones de la complejidad e interconexión entre las distintas comunidades naturales (Berlow et al., 2004; Montoya et al., 2003; Montoya; Solé, 2002; Montoya; Solé, 2003).

Las redes también pueden cla- sificarse según su tamaño (Börner et al., 2007):

\subsection{Redes pequeñas}

Contiene un máximo de 100 nodos. Ejemplos son algunas redes sociales, de ecosistemas biológicos o de exportación-importación de productos entre países. En ellas es posible mostrar la totalidad de nodos, de sus atributos y de los enlaces que les unen. El tamaño de los nodos suele representar atributos como la importancia, el poder o el nivel de trabajo.

\subsection{Redes de tamaño medio}

Incluyen más de 100 y hasta 1.000 nodos. Ejemplos destacados son las redes genéticas, las metabólicas o las económicas, y algunos tipos de redes científicas. En ellas también es posible representar todos sus nodos, pero no todos sus

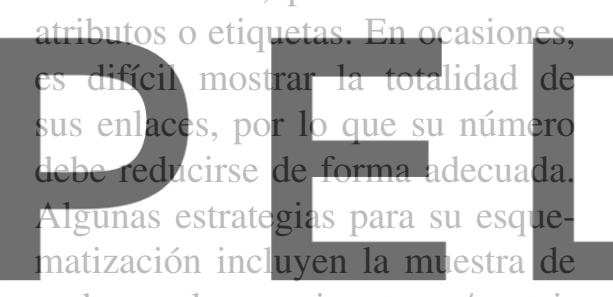
nodos, enlaces etiquetas $y / 0$ atributios destacedos da witilizacióthe vers metáforas visuales (colores...) o la inclusión de sistemas de referencia con ayudas para la navegación.

\subsection{Grandes redes}

Presentan más de 1.000 nodos, como internet, las redes telefónicas, las redes de transportes o de carreteras, y algunas redes científicas, entre otras. Algunos de los principales retos que presenta la esquematización de visualizaciones de este tipo de redes son: la extracción de nodos, enlaces y subgrafos que componen su columna vertebral; la poda de enlaces que evite la pérdida de información relevante; el etiquetado adaptado; y el diseño de interacciones sencillas que faciliten la navegación.

\section{Modelos de redes}

La teoría de grafos surgida a partir de los trabajos de Leonhard
Euler puso por primera vez de manifiesto que la construcción de grafos es la clave para estudiar y comprender mejor el mundo complejo que nos rodea. Pequeños cambios en la topología, aunque afecten sólo a unos pocos nodos o enlaces, pueden proporcionar nuevas e insospechadas posibilidades (Barabási, 2002). A continuación se realizará un recorrido por las propiedades más destacadas de las redes y su evolución desde los inicios de su análisis hasta la actualidad.

\subsection{Redes aleatorias}

Desde hace más de 40 años, la ciencia ha tratado a todas las redes complejas como si fueran puramente aleatorias. Este paradigma fue establecido a partir de los trabajos de los matemáticos húngaros Paul Erdös y Alfréd Rényi, que en 1959 sugirieron que estos sistemas podiar modelarse eficazmente interconectando su
enlaces distribui
importante predi
de redes aleatori

los enlaces de una red hayan sido diştribuidos al azar, el sistema reversigntewithout the watermark co y, por tanto, el número de enlaces por nodo se atiene a una distribución acampanada de Poisson, en la que resulta extraño hallar nodos que cuenten con números mucho mayores o menores de enlaces que la media (Barabási; Bonabeau, 2003; Erdös; Reyni, 1959).

\subsection{Mundos pequeños}

Uno de los experimentos más conocidos sobre mundos pequeños es el del psicólogo Stanley Milgram, que trataba de explicar el concepto de los "seis grados de separación”, basado en la idea de que el número de conocidos de una persona crece exponencialmente con el número de enlaces de la red personal (Milgram, 1963). O también, que todos estamos conectados a través de un máximo de seis contactos intermedios. 
Por su parte, Watts y Strogatz mostraron que si un pequeño número de enlaces es aleatoriamente redirigido en una red aleatoria, ésta se convierte en una red de mundo pequeño, conservando un agrupamiento muy elevado y con cortas distancias entre pares de nodos (Barabási, 2002; Björneborn, 2004; Björneborn, 2006; Watts; Strogatz, 1998).

Esta predicción ha sido confirmada en gran número de las redes estudiadas en distintas disciplinas, $\mathrm{y}$ ha desencadenado diversos trabajos dirigidos no sólo a redes de mundo pequeño sino también al análisis de los principios dinámicos y topológicos de las redes complejas en general. Muchos son los análisis que han caracterizado a las redes del mundo real como mundos pequeños (Albert; Barabási, 2002; Barabási, 2002; Björneborn, 2004;

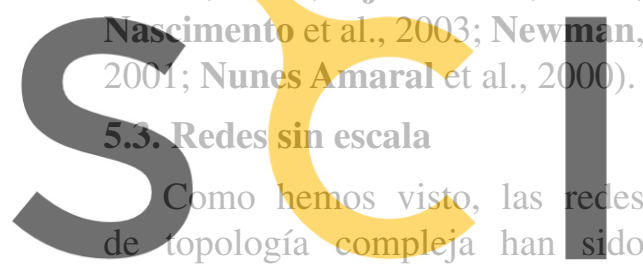

tradicionalmente descritas a partir

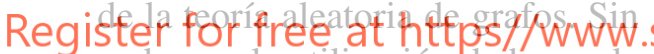
embargo, la utilización de bases de datos y el análisis de los distintos tipos de redes, han permitido concluir que, con independencia del sistema y las entidades que lo constituyen, la probabilidad de que un nodo de la red interactúe con otros desciende siguiendo una curva de ley potencial (power law). Este hallazgo confirma que muchas redes del mundo real se caracterizan por la inexistencia de un patrón que defina el grado de conexión. La distribución de grado en este tipo de redes muestra largas colas, lo que implica que sólo un pequeño número de nodos es conectado por muchos de los otros.

Hay dos aspectos de los patrones de Erdös-Rényi y de WattsStrogatz que no se cumplen en las redes reales. El primero es que ambos patrones asumen que las redes parten de un número fijo de nodos conectados aleatoriamente. Por el contrario, la mayor parte de las redes del mundo real son abiertas y se forman por la adición de nuevos nodos al sistema, por lo que el número de nodos crece durante toda la existencia de la red. En segundo lugar, los patrones aleatorios presuponen que la probabilidad de que dos nodos se conecten es aleatoria y uniforme. Sin embargo, la mayor parte de las redes reales muestran preferencias a la hora de conectarse (Barabási et al., 1999; Barabási; Albert, 1999; Nunes Amaral et al., 2000).

\section{Evolución y dinámica de redes}

En el apartado anterior se han expuesto las dos condiciones de partida de las redes aleatorias: la primera es la asunción de un número

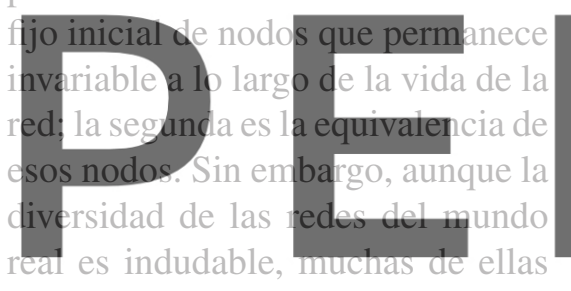

tienen un rasgo en común. Frente al

scipediascara topdowiploadthe des aleatorias, las reales crecen.

Entre los primeros análisis que comprobaron la existencia de redes sin escala se encuentran los trabajos de Derek de Solla Price sobre las redes de citas de trabajos científicos. Encontró que la frecuencia de citas recibidas se distribuye siguiendo una distribución de ley potencial, es decir, la cantidad de citas obtenidas aumenta en proporción a las que ya tienes. El mecanismo de ventaja acumulativa (cumulative advantage) de Price es actualmente aceptado como la explicación más probable a la distribución de grado siguiendo una ley potencial observada en un gran número de redes del mundo real (Price, 1965; Price, 1976).

Otro rasgo que distingue a las redes reales es que en muchas de ellas los nodos tienen una vida limitada (redes sociales) o una capacidad limitada de mantenimiento de enlaces (redes de energía o de ordenadores). Diversos trabajos han demostrado que la edad de los nodos afecta a la hora de conseguir nuevos enlaces, haciéndoles perder su habilidad para la atracción, hecho que aunque no rompe la ley potencial, reduce la inclinación de su ángulo (Albert; Barabási, 2002; Dorogovtsev; Mendes, 2003; Nunes Amaral et al., 2000).

Un último aspecto que debe tenerse en cuenta a la hora de estudiar la evolución de las redes es la lucha por la supremacía en el seno de la red. En la mayor parte de las redes la competición interna por atraer nuevos enlaces no implica un impacto sobre la topología de la red. Sin embargo, en otras el ganador puede atraer hacia sí todos los enla-

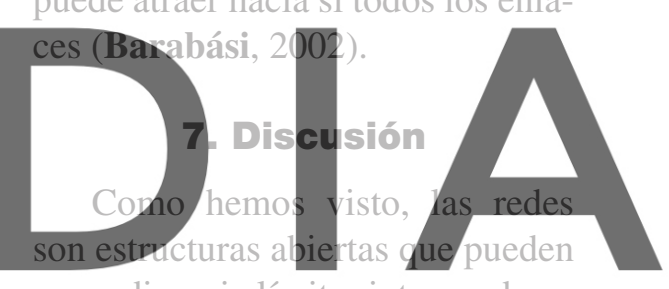

expandirse sin límites integrando en susers nuevernodis en función de
las posibilidades de comunicacion que existan en su entorno y siempre que compartan códigos de comunicación compatibles.

Afortunadamente, son cada vez más abundantes los estudios que se realizan siguiendo esta metodología. En el apartado dedicado a los tipos de redes según los datos empleados para su elaboración, se ha puesto de manifiesto que son dos los tipos de redes de aplicación en nuestra área, las de tipo social y las de información. Pero el análisis de redes es complejo y requiere la asunción de ciertas precauciones, puesto que muchos de esos trabajos adolecen de errores: es frecuente encontrar redes de información caracterizadas como redes sociales o redes no dirigidas analizadas como si de redes dirigidas se tratase. Estos ejemplos ilustran una moda más 
que una corriente, enfocada exclusivamente a la mera representación topológica de grafos. Sin embargo, el análisis estructural va más allá de la visualización que facilita la abstracción del usuario sobre la representación creada, que además, sólo será útil en redes de pequeño tamaño o en grandes redes debidamente esquematizadas y siguiendo los dictados necesarios para su correcta legibilidad.

Este trabajo ha expuesto y enfatizado algunos de los conceptos esenciales de la teoría de redes que deben ser tenidos sistemáticamente en cuenta a la hora de abordar un trabajo relacionado con estudios estructurales, bien desde una perspectiva meramente topológica, o bien desde un análisis de la estructura en su conjunto. No todos ellos, aun formando parte de la literatura corriente en la materia y siendo co-

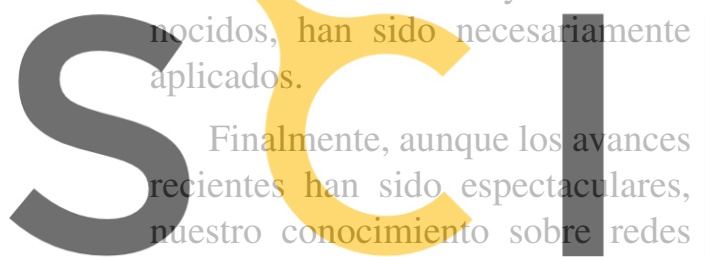
sigue siendo incompleto, abrien-

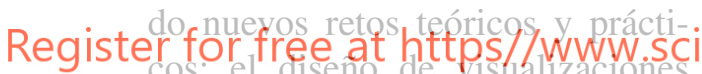
capaces de mostrar sus estructuras y dinámicas o la forma de interaccionar con ellas; las distribuciones de indicadores estructurales; o la comparación de valores nodales, de componentes o de redes completas dirigidas a la exploración de las dinámicas que tienen lugar en el seno de estos sistemas complejos, se presentan como líneas de actuación futuras, susceptibles de convertirse en herramientas muy valiosas como complemento a los estudios tradicionales, con independencia del tamaño de la red.

\section{Bibliografía}

Albert, R.; Barabási, A. L. "Statistical mechanics of complex networks". En: Reviews of modern physics, 2002, 74 (1), p. 47-97.

Barabási, A. L. Linked: the new science of networks. Cambridge: Perseus, 2002
Barabási, A. L.; Albert, R.; Jeong, H. "Meanfield theory for scale-free random networks". En: Physica A, 1999, 272, p. 173-187.

Barabási, A. L.; Bonabeau, E. "Redes sin escala". En: Investigación y ciencia, 2003, julio, p. 58-67.

Barabási, A. L.; Albert, R. "Emergence of scal(5439), p. 509-512.

Barrat, A.; Barthélemy, M.; Pastor-Satorras, R. et al. "The architecture of complex weighted networks". En: Proceedings of the National Academy of Sciences of the United States of America, 2004, 101 (11), p. 3747-3752.

Berlow, E. L.; Neutel, A. M.; Cohen, J. E. et al "Interaction strengths in food webs: issues and opportunities". En: Journal of animal ecology 2004,73 , p. $585-598$.

Björneborn, L. "Mini small worlds of shortest link paths crossing domain boundaries in an academic web space". En: Scientometrics, 2006, 68 (3), p. 395-414

Björneborn, L. "Small-world link structures across an academic web space: a library and information science approach". [Tesis Doctoral]. Copenhagen: Royal School of Library and Information Science, 2004

Börner, K.; Dall'Asta, L.; Ke, W. et al. "Studying the emerging global brain: analyzing and ing in random networks". En: Science, 1999, 286

Moreno, J. L. "Who shall survive? A new approach to the problem of human interrelations". Washington: Nervous and Mental Disease, 1934.

Moreno, J. L. "Who shall survive? Foundations of sociometry, group psychotherapy and sociodrama”. New York: Beacon House, 1953.

Nascimento, M. A.; Sander, J.; Pound, J. "Analysis of Sigmod's co-authorship graph". En: ACM Sigmod record, 2003, 32 (3), p. 8-10.

Newman, M. E. J. "The structure of scientific collaboration networks". En: Proceedings of the National Academy of Sciences of the United States of America, 2001, 98 (2), p. 404-409.

Newman, M. E. J. "The structure and function of complex networks". En: SIAM review, 2003. 45 (2), p. 167-256.

Nunes Amaral, L. A.; Scala, A.; Barthélemy, M. et al. "Classes of small-world networks". En: Proceedings of the National Academy of Sciences of the United States of America, 2000, 97 (21). p. 11149-11152

Otte, E.; Rousseau, R. "Social network analysis: a powerful strategy, also for the information sciences". En: Journal of information science, 2002, 28 (6), p. 441-453

Perianes-Rodríguez, A.; Olmeda-Gómez, C. Moya-Anegón, F. "Detecting research groups in coauthorship networks". En: Collnet meeting, 90,
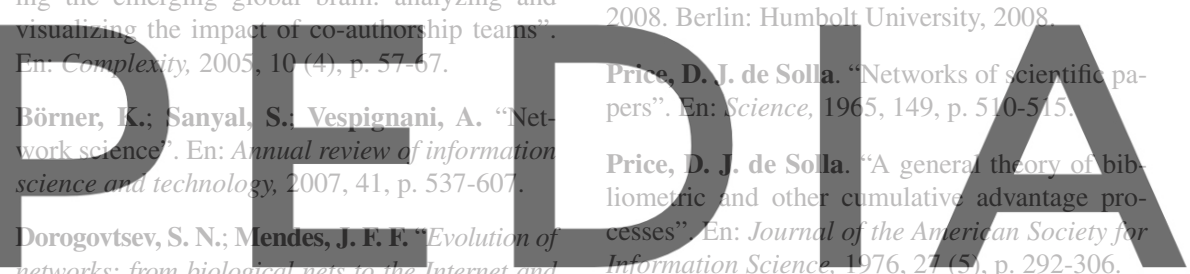

WWW". Oxford: Oxford University Press, 2003.

pedia.comito dowatoad the: Publications mathematicae, 1959, 6, p. 290-297.

Faba Pérez, C.; Guerrero-Bote, V. P.; MoyaAnegón, F. "Self-organizing maps of Web spaces based on formal characteristics". En: Information processing and management, 2005, 41 (2), p. 331-346.

Faba Pérez, C.; Guerrero-Bote, V. P.; MoyaAnegón, F. "Fundamentos y técnicas cibermétricas”. Mérida: Junta de Extremadura, 2004.

Milgram, S. "Behavioral study of obedience". En: Journal of abnormal and social psychology, 1963, 67 (4), p. 371-378.

Molina, J. L. "El análisis de redes sociales: una introducción”. Barcelona: Bellaterra, 2001. (Serie general universitaria, 10).

Montoya, J. M.; Rodríguez, M. A.; Hawkins, B. A. "Food web complexity and higher-level ecosystem services". En: Ecology letters, 2003, 6, p. 587-593.

Montoya, J. M.; Solé, R. V. "Small world patterns in food webs". En: Journal of theoretical biology, 2002, 214, p. 405-412.

Montoya, J. M.; Solé, R. V. “Topological properties of food webs: from real data to community assembly models". En: Oikos, 2003, 102 (3), p. 614-622.

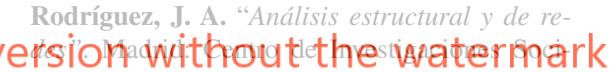

\section{ologicas, 1995. (Cuadernos metodologices, 16).}

Vargas-Quesada, B; Moya-Anegón, F. "Visualizing the structure of science”. Berlin: Springer, 2007.

Watts, D. J.; Strogatz, S. H. A. "Collective dynamics of small-world networks". En: Nature, 1998, 393, p. 440-442.

\section{Antonio Perianes-Rodríguez y Car- los Olmeda-Gómez}

Departamento de Biblioteconomía y Documentación. Universidad Carlos III de Madrid, C/ Madrid, 128, 28903 Getafe (Madrid), España. antonio.perianes@uc3m.es carlos.olmeda@uc3m.es

\section{Félix de Moya-Anegón}

Profesor de Investigación, Consejo Superior de Investigaciones Científicas (CSIC), Madrid, España felix@ugr.es 